\title{
Prevalencia de estomatitis subprotésica asociada a candida albicans en pacientes portadores de prótesis total superior en asilos del valle de los Chillos, Ecuador.
}

\section{Prevalence of subprosthetic stomatitis associated with candida albicans in patients with complete overdentures in nursing homes at the valle de los Chillos, Ecuador.}

\author{
Verónica Mosquera-Cisneros ${ }^{1}$, María Gabriela Romero ${ }^{1}$, Andrés Viteri-García ${ }^{2}$ y Paula Zambrano-Achig Z $^{2}$ \\ ${ }^{1}$ Facultad de Odontología, Universidad de Las Américas (UDLA). Quito, Ecuador. \\ ${ }^{2}$ Universidad UTE. Centro de Investigación de Salud Pública y Epidemiología Clínica (CISPEC). Quito, Ecuador. \\ *paula.zambrano@ute.edu.ec
}

\begin{abstract}
Resumen
Objetivo: Determinar la prevalencia de estomatitis subprotésica asociada a Candida albicans en pacientes portadores de prótesis total superior removible, en centros de atención geriátrica (asilos) del Valle de Los Chillos, Ecuador. Metodología: Estudio trasversal descriptivo, se consideró como población de estudio a todos los pacientes portadores de prótesis total superior removible $(n=79)$ de siete asilos ubicados en el Valle de Los Chillos; se realizó el examen clínico intraoral para evaluar la presencia de estomatitis subprotésica, el estado de adaptación de la prótesis, el pH palatino, el índice de placa de las prótesis y una muestra microbiológica palatina. Se recolectó los datos en una ficha numerada y se procesaron en el paquete estadístico SPSS v22. Resultados: el $41 \%(\mathrm{n}=32)$ de los pacientes presentó signos de estomatitis subprotésica. De estos pacientes el $68.8 \%(\mathrm{n}=22)$ presentaron estomatitis subprotésica asociada a Candida albicans y pH palatino ácido. De los 22 pacientes el 43,8\% $(n=10)$ presentaron un índice de placa alto al momento de la evaluación y también 9,4\% (n=2) de los pacientes presento desadaptación de la prótesis total superior removible. Conclusiones: Existe alta prevalencia de estomatitis subprotésica asociada a Candida albicans en asilos del Valle de Los Chillos. La presencia de Candida albicans tiene relación con pH palatino ácido y mala higiene de la prótesis, mientras que no se relaciona con el estado de desadaptación de la prótesis. Es importante recalcar que estos últimos datos se deben tomar con precaución, ya que la mala adaptación protésica puede causar daños a los tejidos y es considerado un factor de riesgo para el desarrollo de estomatitis subprotésica
\end{abstract}

Palabras clave: Estomatitis subprotética, Candida albicans, Dentadura Completa Superior.

Objective: To determine the prevalence of subprosthetic stomatitis associated with Candida albicans in patients with removable complete prostheses, in nursing homes at the Valle de Los Chillos, Ecuador. Methodology: Descriptive crosssectional study; the study population included patients with removable complete prostheses $(n=79)$ from seven nursing homes located at the Valle de Los Chillos. An intra-oral clinical examination was performed to evaluate the presence of subprosthetic stomatitis, adaptation of the prosthesis to the palate, palate's $\mathrm{pH}$ levels, prosthesis' plaque index and to obtain a microbiological sample from the palate. Data was collected on a register and analysed with the SPSS v22 statistical package. Results: $41 \%(n=32)$ patients were diagnosed with subprosthetic stomatitis. Out of these 32 patients, $68,8 \%(n=22)$ presented subprothetic stomatitis associated with Candida albicans and an acidic palate's. Out of the 22 patients, 43.8\% (n $=10)$ had a high plaque index at the time of evaluation and $9.4 \%(n=2)$ of the patients had a mismatch of the removable complete prosthesis. Conclusions: This study shows that there is a high prevalence of subprosthetic stomatitis associated with Candida albicans in patients from nursing homes at the Valle de Los Chillos. Furthermore, the presence of Candida albicans was shown to be associated with an acidic palate's $\mathrm{pH}$ and poor hygiene of the prosthesis, its presence was not associated with the adaptation of the prosthesis to the palate. It is important to emphasize that these last data should be taken with caution, since prosthetic maladaptation can cause tissue damage and is considered a risk factor for the development of subprosthetic stomatitis

Key words: Stomatitis, Denture; candida albicans, Denture, Complete, Upper. 


\section{Introducción}

Existen diversas opciones de tratamiento para rehabilitar a los pacientes edéntulos totales; ${ }^{1,2}$ entre las más populares tenemos los implantes, los cuales ofrecen los mejores beneficios al paciente tanto en comodidad como en estética, ${ }^{3}$ pero no todos los pacientes tienen acceso a este tipo de tratamiento y es por esto, por lo que las prótesis totales (sobredentaduras) aún siguen siendo la opción de tratamiento mayormente utilizada para este tipo de pacientes, ${ }^{4}$ por lo que es importante conocer ciertas complicaciones que se pueden generar por su uso.

La estomatitis subprotésica es una complicación del uso inadecuado de la prótesis total superior removible, es considerada una inflamación crónica visible como un enrojecimiento de la mucosa oral, especialmente presenta mayor afección en el maxilar superior en la zona del paladar. ${ }^{5,6}$

En Ecuador se han realizado estudios sobre estomatitis subprotésica en clínicas docentes universitarias de las ciudades de Quito, Guayaquil y Cuenca y se ha encontrado que la prevalencia es superior a $50 \%$ en los pacientes adultos portadores de prótesis totales que acuden a estos servicios, ${ }^{7-10}$ en la región existen reportes que mencionan que la prevalencia va desde el $50 \%$ hasta $60 \%$ en portadores de prótesis. ${ }^{11-13}$

Diferentes factores de riesgo han sido descritos para el desarrollo de estomatitis, entre ellos se encuentran: prótesis desadaptadas, higiene de la prótesis y de la boca deficientes, uso continuo y prolongado de la prótesis, condiciones sistémicas como diabetes e inmunodeficiencias, hipersensibilidad a los materiales de la base de la prótesis y tabaquismo ${ }^{14,15}$

Varios autores afirman que existe un impacto significativo entre una higiene oral deficiente y el desarrollo de estomatitis subprotésica, uno de los microorganismos que con mayor frecuencia se asocia a esta patología es la Candida albicans. ${ }^{16,17}$ Por lo general esta patología es asintomática, pocos pacientes presentan sensación de ardor en la boca al momento de ingerir alimentos y es muy raro que se produzca disfagia. $^{18}$

La mayoría de los pacientes portadores de prótesis consideran que las irritaciones palatinas que presentan son consecuencias normales del uso de la prótesis, desconocen que ésta puede ser causada por la presencia de microorganismos y no le prestan la debida importancia. ${ }^{19,20}$

El objetivo de esta investigación fue estudiar prevalencia de estomatitis subprotésica asociada a Candida albicans en pacientes portadores de prótesis total superior removible, en centros de atención geriátrica (asilos) del Valle de Los Chillos, Ecuador.

\section{Métodos}

Se planteó un estudio transversal descriptivo. Se analizó a la población total de 246 adultos mayores que residen en siete asilos del Valle de Los Chillos (118 hombres y 128 mujeres), de los cuales 79 pacientes eran portadores de prótesis total superior removible. El período de reclutamiento y recolección de datos se realizó entre el 01 de octubre y 01 de noviembre de 2015, se contó con la aprobación de las autoridades de las instituciones de los asilos de ancianos y el consentimiento informado de todos los participantes

Se realizó una anamnesis y examen intraoral a todos los participantes, estos datos fueron recogidos en una historia clínica específicamente diseñada para este estudio. Para el diagnóstico y clasificación de la estomatitis subprotésica se utilizó la escala de Newton, dicha clasificación se describe de la siguiente manera: "Grado I: lesiones clínicas caracterizadas por signos inflamatorios mínimos, generalmente asintomáticos. Pueden aparecer áreas hiperémicas localizadas o en forma de pequeños puntos eritematosos. Grado II: lesiones francamente inflamatorias, pueden llegar a observarse los contornos de la prótesis. La superficie mucosa es roja brillante, aparecen áreas eritematosas difusas. Grado III: lesiones constituidas por una mucosa gruesa de gránulos irregulares, que pueden tener aspecto aterciopelado o papilar". ${ }^{21}$ Los hallazgos clínicos fueron documentados a través de fotografías, las cuales fueron anexadas a la historia clínica.

Para el estado de adaptación protésico se realizó un análisis visual mediante la evaluación clínica.

El pH salival fue valorado a tempranas horas de la mañana, antes del desayuno, con tiras reactivas colocadas en la mucosa palatina. Estas fueron sostenidas con la lengua del paciente contra el paladar por 10 segundos.

La higiene de la prótesis fue evaluada mediante la tinción de violeta de genciana al $1 \%$, el proceso de evaluación fue el siguiente: la prótesis dental de los pacientes fue retirada y sumergida en agua por 10 segundos, una vez retirada del agua, sobre la prótesis se colocó violeta de genciana al $1 \%$, se sumergió nuevamente la prótesis en agua y al retirarla se evaluó las zonas de la prótesis que se encontraron coloreadas por la tinción.

Para la identificación de Cándida albicans se realizó un frotis en paladar, posteriormente se colocó la muestra en medio Cary-Blair y fue enviada por medio de transporte Stuart al Laboratorio de Microbiología para ser analizada. En el laboratorio se realizó el aislamiento e identificación de Candida albicans mediante dos técnicas de laboratorio (Agar Biggy y prueba del tubo germinal). ${ }^{22,23}$

Los datos de adaptación de la prótesis, prueba microbiológica, pH y evaluación de placa bacteriana fueron ingresados a la base de datos del programa estadístico SPSS versión 22, con el propósito de obtener estadígrafos descriptivos de frecuencia.

\section{Resultados}

Siete asilos del Valle de Los Chillos fueron tomados en cuenta para la presente investigación, de los cuales 79 pacientes cumplieron con los criterios de inclusión y exclusión. De estos, $41 \%(n=32)$ presentaron signos de estomatitis subpro- 
tésica; de estos el, $68.8 \%(\mathrm{n}=22)$ presentaron un diagnóstico positivo de Candida albicans.

De los pacientes que presentaron estomatitis subprotésica, el $21.9 \%(\mathrm{n}=7)$ presentó desadaptación de la prótesis. Con respecto al $\mathrm{pH}$ palatino el $65,6 \%(\mathrm{n}=21)$ presentó un $\mathrm{pH}$ ácido. Se encontró que según la escala de Newton el 81,3\% $(n=26)$ presentaron grado I y $18,8 \%(n=6)$ grado II. Para el índice de placa, un $50 \%(\mathrm{n}=16)$ presentó índice malo.

Respecto a las variables asociadas a Candida albicans, el $77.2 \%(n=17)$ de los pacientes diagnosticados con Candida albicans presentaron $\mathrm{pH}$ palatino ácido.

La asociación entre el mal índice de placa, estado de adaptación de la prótesis y Cándida albicans fue de 63,6\% $(\mathrm{n}=3)(\mathrm{p}=0,002), 13,6 \%(\mathrm{n}=3)(\mathrm{p}=0,09)$ respectivamente $($ Tabla1).

Tabla 1. Índice de placa, estado de adaptación de la protesis y Candida albicans

\begin{tabular}{lccc}
\hline & & \multicolumn{2}{c}{ Positivo } \\
Asociación & Indice & $\mathrm{N}(22)$ & $100 \%$ \\
\hline Mal indice de placa & Satisfecho & 8 & $36,4 \%$ \\
& Malo & 14 & $63,6 \%$ \\
\hline $\begin{array}{l}\text { Estado de adaptac- } \\
\text { cion de protesis }\end{array}$ & Adaptada & 19 & $86,4 \%$ \\
& Desadaptada & 3 & $13,6 \%$ \\
\hline
\end{tabular}

Con respecto a la asociación entre la mala adaptación de la prótesis y Candida albicans los resultados no son estadísticamente significativos. Pero debemos manejar estos datos con precaución, ya que la mala adaptación de la prótesis puede causar lesiones en la mucosa y dichas lesiones pueden convertirse en un factor de riesgo que acelere el desarrollo de la estomatitis subprotésica.

\section{Discusion}

Los resultados de esta investigación concuerdan parcialmente con los presentados en la literatura respecto a la prevalencia de estomatitis subprotésica, en esta investigación se encontró un $41 \%(\mathrm{n}=32)$ de prevalencia en los adultos mayores que se encuentran en los asilos de ancianos, en diferentes estudios realizados en Ecuador se encontró una prevalencia de varía entre $52 \%$ hasta $70 \%$ en población adulta y adultos mayores. $^{7-10}$

El estudio determinó que la presencia de Candida albicans fue de $68.8 \%(n=22)$ y esto concuerda con diferentes estudios que atribuyen que dicho microorganismo es un factor que ayuda a agravar el cuadro y que llegue a etapas crónicas con mayor rapidez, en estos estudios la asociación de estomatitis subprotésica con Candida albicans fue de un $60 \%$ de los pacientes. ${ }^{24,25}$.

Existen diferentes factores que pueden interactuar para el desarrollo de la estomatitis subprotésica como la higiene de la prótesis y la cavidad oral. En diferentes estudios se ha reportado que la higiene de la prótesis es un factor de riesgo, pudiendo influir en un $40 \%$ en la presencia de la enfermedad. ${ }^{26}$ En este estudio el $50 \%$ de la población presenta una mala higiene, esto puede deberse a la frecuencia del uso de la prótesis y al material de esta. ${ }^{27}$ Se demostró que existe una relación de $63,6 \%$ de presencia de C. albicans en los pacientes con mala higiene de la prótesis. Algunos autores coinciden en sus estudios con esta asociación que hay mayor disposición de Candida albicans en prótesis que presentan pobre higiene. ${ }^{26}$ la cantidad de placa bacteriana y el tiempo que se encuentre esta en contacto con la mucosa palatina puede ser el factor de riesgo de mayor presencia de Candida albicans. ${ }^{26,28}$

Con respecto al $\mathrm{pH}$ palatino en este estudio el 65,6\% presentó un $\mathrm{pH}$ ácido esto concuerda con la literatura ya que se ha reportado $\mathrm{pH}$ ácidos en el $50 \%$ de la población estudiada. ${ }^{29,30}$ La relación entre el $\mathrm{pH}$ palatino ácido y Candida albicans en este estudio fue de $77,2 \%$ esto se puede deber a que el $\mathrm{pH}$ ácido promueve la adherencia de la Candida albicans aumentando la probabilidad de que la enfermedad se desarrolle. ${ }^{24}$

La desadaptación de la prótesis se presentó en un 13,6\% con relación a la Candida albicans. A pesar de que la comparación resultó no ser estadísticamente significativa en este estudio, hay que tomar estos datos con precaución ya que la mala adaptación protésica puede causar daño en los tejidos y por lo tanto puede ser un factor de riesgo para el desarrollo de estomatitis subprotésica con o sin C. albicans. ${ }^{31,32}$

Es importante conocer que el cambio de prótesis o la mejora de la prótesis antigua no resolverá nada, sin antes a ver eliminado de raíz el problema que causa la patología. Esto solo ocultara por varios meses el problema, pero se ha demostrado que la Candida albicans puede colonizar las superficies de las prótesis y producir una lesión crónica. Por esta razón no se recomienda realizar rebases de acrílico sin antes a ver tratado la patología directamente. ${ }^{31,32}$

La principal fortaleza de este estudio es que se incluyó a toda la población de los asilos del Valle de Los Chillos. Algunas de las limitaciones de este estudio fueron que, debido al diseño propuesto, no es posible comparar entre grupos de interés (por ejemplo, adultos mayores internados versus no internados, o diferentes patologías relacionadas a la prótesis total superior removible). El uso de las tiras reactivas para determinar el $\mathrm{pH}$ no es método más adecuado para medir el $\mathrm{pH}$ bucal.

Es necesario realizar un control odontológico rutinario en los asilos del Valle de Los Chillos, Pichincha - Ecuador. Los odontólogos que asistan a asilos de ancianos deben estar preparados para la detección y tratamiento de estomatitis subprotésica debido a su alta prevalencia. Futuros estudios con diseños prospectivos deberían evaluar (las variables) en todos los grupos relevantes, para analizar si esta situación se replica en los ancianos que no se encuentran internados en asilos. 


\section{Conclusiones}

Se encontró una alta prevalencia de estomatitis subprotésica asociada a Candida albicans en asilos del Valle de Los Chillos, Ecuador. Además, se observó una relación entre la mala higiene de la prótesis, el ph acido palatino como un factor de riesgo para el desarrollo de estomatitis subprotésica asociada a Candida albicans. Por estas razones antes mencionadas es importante dar a conocer estos resultados, para mejorar la higiene de los adultos mayores que se encuentran dentro de estos centros de cuidado y considerar realizar consultas odontológicas periódicas para evitar y mejorar el estado de salud bucal de nuestros adultos mayores

\section{Financiamiento}

Este estudio fue realizado con financiamiento propio.

\section{Conflicto de Intereses}

Los autores declaran no presentar ningún conflicto de intereses en relación con el tema de estudio.

\section{Agradecimiento}

Los autores desean agradecer la participación de los pacientes de los asilos San Juan de Dios, Años Dorados, Copitos de nieve, Dulce María, Mi Amigo Divino, Hogar de Vida 1 y Mi Amigo Divino Club. La Od. Verónica Mosquera Cisneros es estudiante del programa de Rehabilitación Oral de la Universidad de las Américas.

\section{Referencias Bibliográficas}

1 Basker RM, Davenport JC, Thomason JM. Prosthetic treatment of the edentulous patient. John Wiley \& Sons; 2011.

2 Felton D, Cooper L, Duqum I, Minsley G, Guckes A, Haug $\mathrm{S}$, et al. Evidence-based guidelines for the care and maintenance of complete dentures: A publication of the American College of Prosthodontists. Journal of Prosthodontics: Implant, Esthetic and Reconstructive Dentistry. 2011;20:S1S12.

3 Brennan M, Houston F, O’Sullivan M, O’Connell B. Patient satisfaction and oral health-related quality of life outcomes of implant overdentures and fixed complete dentures. International Journal of Oral \& Maxillofacial Implants. 2010;25(4).

4 Ayuso-Montero R, Martori López E, Brufau de Barberà M, Ribera Uribe M. Prótesis removible en el paciente geriátrico. Avances en Odontoestomatología. 2015;31(3):191-201.

5 Wilson J. The aetiology, diagnosis and management of denture stomatitis. British dental journal. 1998;185(8):380 384.

6 Gendreau L, Loewy ZG. Epidemiology and etiology of denture stomatitis. Journal of Prosthodontics: Implant, Esthetic and Reconstructive Dentistry. 2011;20(4):251-260.
7 Holguin Limones AD. Prevalencia de estomatitis subprotésica. [B.S. thesis]. Universidad de Guayaquil. Facultad Piloto de Odontología; 2019.

8 Flores Enríquez AE. Prevalencia de estomatitis subprotésica en la mucosa bucal de pacientes con prótesis total superior pertenecientes al Centro de Referencia Manuela Sáenz periodo 2016 [B.S. thesis]. Quito: UCE; 2017.

9 Pachar Nicole CE. Prevalencia de estomatitis subprotésica en pacientes portadores de prótesis removibles totales y parciales que fueron atendidos en la Facultad de Odontología de la Universidad de Cuenca en el periodo 2012 - 2016. [B.S. thesis]. Universidad de Cuenca; 2016.

10 Tucta Céspedes TK. Prevalencia de Estomatitis por uso de prótesis total mal adaptada en pacientes atendidos en clínica UCSG. 2016;.

11 Lorduy MC, Rozo MP, Blanco KA, Marquez KB, Múnera YC. Presencia de Candida en pacientes con estomatitis subprotesica que acuden a la facultad de odontología de la universidad de Cartagena en el periodo transcurrido entre agosto 2006-junio 2007. Duazary. 2008;5(2):90-98.

12 Espinoza I, Rojas R, Aranda W, Gamonal J. Prevalence of oral mucosal lesions in elderly people in Santiago, Chile. Journal of oral pathology \& medicine. 2003;32(10):571575.

13 Pires F, Santos E, Bonan P, De Almeida O, Lopes M. Denture stomatitis and salivary Candida in Brazilian edentulous patients. Journal of oral rehabilitation. 2002;29(11):11151119.

14 Aoun G, Cassia A. Evaluation of denture-related factors predisposing to denture stomatitis in a Lebanese population. Materia socio-medica. 2016;28(5):392.

15 Yarborough A, Cooper L, Duqum I, Mendonça G, McGraw $\mathrm{K}$, Stoner L. Evidence regarding the treatment of denture stomatitis. Journal of prosthodontics. 2016;25(4):288-301.

16 Otero Rey E, Peñamaría Mallón M, Rodríguez Piñón M, Martín Biedma B, Blanco Carrión A. Candidiasis oral en el paciente mayor. Avances en odontoestomatología. 2015;31(3):135-148.

17 VASCONCELOS LCd, VASCONCELOS LCdS, GHERSEL ELdA, VELOSO DJ, CUNHA PÂSMdA. Denture hygiene: importance in denture stomatitis control. RGO Revista Gaúcha de Odontologia (Online). 2013;61(2):255261.

18 Maciąg J, Osmenda G, Nowakowski D, Wilk G, Maciąg A, Mikołajczyk T, et al. Denture-related stomatitis is associated with endothelial dysfunction. BioMed Research International. 2014;2014.

19 Pattanaik S, Vikas B, Pattanaik B, Sahu S, Lodam S. Denture Stomatitis: A literature review. Journal of Indian Academy of Oral Medicine and Radiology. 2010;22(3):136.

20 de Vasconcellos AA, Gonçalves LM, Cury AADB, da Silva WJ. Candida-associated denture stomatitis: Clinical relevant aspects. In: Oral Candidosis. Springer; 2015. p. 53-57. 21 AV N. Denture sore mouth. A possible etiology. Br Dent 
$\mathrm{J} ; 112(35): 57-60$.

22 Khadka S, Regmi P, Giri S, Shah PK, Mishra SK. Identification of Candida species using CHROM agar. International Journal of Medicine and Biomedical Sciences. 2016;1(3):10-13.

23 Matare T, Nziramasanga P, Gwanzura L, Robertson V. Experimental germ tube induction in Candida albicans: An evaluation of the effect of sodium bicarbonate on morphogenesis and comparison with pooled human serum. BioMed 30 research international. 2017;2017.

24 Marinoski J, Bokor-Bratić M, Čanković M. Is denture stomatitis always related with candida infection? A case 31 control study. Medicinski Glasnik. 2014;11(2).

25 BIANCHI CMPdC, Bianchi HA, Tadano T, PAULA CRd, 32 Hoffmann-Santos HD, Leite Jr DP, et al. Factors related to oral candidiasis in elderly users and non-users of removable dental prostheses. Revista do Instituto de Medicina Tropical de São Paulo. 2016;58.

26 Gacon I, Loster JE, Wieczorek A. Relationship between oral hygiene and fungal growth in patients: users of an acrylic denture without signs of inflammatory process. Clinical Interventions in Aging. 2019;14:1297.

27 Evren BA, Uludamar A, Işeri U, Ozkan YK. The association between socioeconomic status, oral hygiene practice, denture stomatitis and oral status in elderly people living different residential homes. Archives of gerontology and geriatrics. 2011;53(3):252-257.
28 Kulak-Ozkan Y, Kazazoglu E, Arikan A. Oral hygiene habits, denture cleanliness, presence of yeasts and stomatitis in elderly people. Journal of oral rehabilitation. 2002;29(3):300-304.

29 Budtz-Jørgensen E, Mojon P, Rentsch A, Deslauriers N. Effects of an oral health program on the occurrence of oral candidosis in a long-term care facility. Community dentistry and oral epidemiology. 2000;28(2):141-149.

30 Shulman J, Rivera-Hidalgo F, Beach M. Risk factors associated with denture stomatitis in the United States. Journal of oral pathology \& medicine. 2005;34(6):340-346.

1 Hadjieva H, Dimova M, Todorov S. Stomatitis prosthetica-a polyetiologic disorder. Journal of IMAB. 2006;12(2):38-4.

Altarawneh S, Bencharit S, Mendoza L, Curran A, Barrow D, Barros S, et al. Clinical and histological findings of denture stomatitis as related to intraoral colonization patterns of Candida albicans, salivary flow, and dry mouth. Journal of Prosthodontics: Implant, Esthetic and Reconstructive Dentistry. 2013;22(1):13-22.

Recibido: 30 de enero del 2020

Aceptado: 01 de agosto del 2020 
